\title{
Trajectory Optimization for Ascent and Glide Phases Using Gauss Pseudospectral Method
}

\author{
Abdel Mageed Mahmoud, Chen Wanchun, Zhou Hao, and Liang Yang
}

\begin{abstract}
The trajectory optimization method for ascent and glide phases' flight of a hypersonic vehicle is studied. In addition to the constraints on the final position coordinates, the final orbit position and velocity are specified. This article presents also the purpose of designing the endpoint and the constraints during the fight until the endpoint. The trajectory optimization is divided into two phases: vertical-rise phase and orbit-insertion phase respectively. The trajectory optimization problem is formulated as the multi-constraints optimal control problem. Gauss Pseudospectral Method (GPM) method, it is converted into the nonlinear programming which is solved by SNOPT. This direct optimization technique uses linkage constraints to enforce state and optimization parameter continuity between phases. The independent phases, linked together, allow for a common cost or objective function to be optimized. The derivative of reference angle of attack is chosen as the control variables, the performance index is the weighted sum of squares of this derivative. Results show that the proposed method able to iteratively provide the solution coming closer to the necessary condition for optimality and terminal constraints.
\end{abstract}

Index Terms-Trajectory optimization, optimal control Multi constraints nonlinear programming.

\section{INTRODUCTION}

Trajectory optimization has been a topic of considerable research of launch vehicles for over 40 years in practical engineering application where the optimal control theory is commonly applied to obtain optimal solutions. "The objective of an optimal control problem is to determine the control signals that will cause a process to satisfy the physical constraints and at the same time minimize (or maximize) some performance index" As defined by Kirk. Possible performance indices include time, fuel consumption, or any other parameter of interest in a given application [1], [2].

Trajectory optimization of hypersonic vehicle is a difficult problem due to various constraints of thermal load, total load and dynamic pressure .Numerous researches are being carried out in this field. Lin Ma, Zhijiang Shao, Weifeng Chen, Xinguang Lv and Zhengyu Song have optimized the problem of fuel-optimal lunar ascent phase using constant-thrust propulsion [3]. Tawifiqur Rahman, Zhou Hao used Legendre and Gauss pseudospectral methods to optimize the trajectory problem of a hypersonic vehicle [4]. Fariba Fahroo and I. Michael Ross solved the problem of Bolza arising in trajectory Optimization [5]. Michael A. Paluszek and Stephanie J. Thomas compared three different global indirect approaches for solving the problem of finding optimal trajectories for low thrust spacecraft[6]. Numerical

Manuscript received August 5, 2016; revised October 12, 2016.

The authors are with School of Astronautics, Beihang University, Beijing, 100191, China (e-mail: wanchun_chen@buaa.edu.cn). algorithms of trajectory optimization for flight vehicles are currently studied. Huang GuoQiang, Lu YuPing and Nan Ying summarized the basic principle, characteristics and application for all kinds of current trajectory optimization algorithms [7].

One of the most general solutions to the optimal control problems is the calculus of variations and Pontryagin's maximum principle. By applying these methods determine the first order necessary conditions for a solution, these necessary conditions reduce the optimal control problem to a two-point boundary value problem. For most problems, the boundary value problem is difficult to solve analytically so numerical techniques are used to determine an approximation to the continuous problem. Numerical methods fall into Direct and Indirect methods [8].

Some of Indirect methods are multiple shooting, quasi-linearization and collocation which approximating the solution to the continuous necessary conditions. The advantages of indirect methods is the high accuracy and emphasis the solution satisfies the necessary optimality conditions but the disadvantage that the necessary optimality conditions must be derived analytically, the convergence is small so a good initial guess is required and also a guess is required for costate, finally for path constrained problems the constrained and unconstrained arcs should know a priori [9].

Direct methods transcribe the continuous optimal control problem into a nonlinear programming problem (NLP), which can be solved by well-developed algorithms. One of the most advantages the optimality conditions do not need to be derived, large radius of convergence, no need for a guess of the costates and finally the switching structure does not need to be known.

Another approach based on spectral methods used to parameterize both the states and controls. Piecewise polynomials used to approximate the differential equations at collocation points, states and controls can also be parameterized using global polynomials which typically have faster convergence rates than traditional methods. This method provides accurate state and control approximations.

Spectral methods were applied to optimal control problems using Chebyshev polynomials and then developed the Legendre pseudospectral method using Lagrange polynomials and collocation at Legendre-Gauss-Lobatto (LGL) points, finally Gauss pseudospectral method (GPM) has been shown to satisfy the optimality conditions for a large class of problems [8], [10].

Gauss Pseudospectral Method (GPM) proved an efficient way to solve the trajectory optimization problem, and beside it has high precision and fast rate of convergence, it equivalence the Karush-kuhun-tucker (KKT) condition and the Hamiltonian Boundary Value Problem (HBVP).

In this paper an algorithm is described to solve 
multiple-phase optimal control problems using a numerical method called the Gauss pseudospectral method. The algorithm discretized the cost functional and the differential-algebraic equations in each phase of the optimal control problem. The phases are then connected using linkage conditions on the state and time.

\section{VEHICLE MODEL}

The state differential equations for the ascent phase without considering the earth rotation and lateral movement are described as follows[11]:

$$
\begin{gathered}
\dot{\mathrm{x}}=v \cos (\gamma) \\
\dot{\mathrm{h}}=v \sin (\gamma) \\
\dot{v}=\frac{T \cos (\alpha)-D}{m}-g \sin (\gamma) \\
\dot{\gamma}=\frac{T \sin (\alpha)+L}{m v}-\frac{g}{v} \cos (\gamma)+\frac{v}{r} \cos (\gamma) \\
\dot{m}=-\frac{T}{I_{s p} g_{0}}
\end{gathered}
$$

where $x, h$ is the down range and height respectively, $\mathrm{v}$ is the velocity vector, $\alpha$ is the angle of attack, $\gamma$ are the flight path angle and $\mathrm{m}$ is the mass, $T$ is the thrust vector, $\mathrm{g}$ is the gravitational force while for the gliding phase adding angle $\psi$ is the azimuth angle to the dynamics equations. $(D, L)$ is the drag and lift force and are given as[12]:

$$
\begin{gathered}
D=\frac{1}{2} \rho v^{2} \mathrm{~S}_{r e f} \mathrm{C}_{D}\left(M_{a}, \alpha\right) \\
L=\frac{1}{2} \rho v^{2} \mathrm{~S}_{r e f} \mathrm{C}_{L}\left(M_{a}, \alpha\right)
\end{gathered}
$$

where $\left(\rho, S_{\text {ref }}\right)$ are the air density of the current altitude and the reference area, respectively, $C_{L}$ and $C_{D}$ are the lift and drag coefficients, respectively, which are the non-linear functions of the attack $\alpha$ angle and the Mach $M_{a}$.

In order to consider the states differential equations for the glide phase, we should convert the previous equations to 3-D differential equation by adding static parameter $\psi$ at the final states of ascent phase as follows:

$$
\begin{aligned}
& M_{x}=\left[\begin{array}{ccc}
1 & 0 & 0 \\
0 & \cos \left(\frac{\pi}{2}-\text { lat }\right) & \sin \left(\frac{\pi}{2}-\text { lat }\right) \\
0 & -\sin \left(\frac{\pi}{2}-\text { lat }\right) & \cos \left(\frac{\pi}{2}-\text { lat }\right)
\end{array}\right] \\
& M_{z}=\left[\begin{array}{ccc}
\cos \left(\text { lon }+\frac{\pi}{2}\right) & \sin \left(\text { lon }+\frac{\pi}{2}\right) & 0 \\
-\sin \left(\text { lon }+\frac{\pi}{2}\right) & \cos \left(\text { lon }+\frac{\pi}{2}\right) & 0 \\
0 & 0 & 1
\end{array}\right]
\end{aligned}
$$

lat is the latitude lon is the longitude

$$
\mathrm{C}_{\mathrm{gs}}=M_{x} * M_{z}
$$

$$
\mathrm{C}_{s g}=\mathrm{C}_{\mathrm{gs}}{ }^{\prime}
$$

$\mathrm{C}_{\mathrm{gs}}$ is the transformation matrix from space to ground $\mathrm{C}_{s g}$ is the transformation matrix from ground to space $R=6378145$

$R$ is the radius of the earth

$$
\begin{gathered}
R_{x_{-} s}=R * \cos (\text { lat }) * \cos (\text { lon }) \\
R_{y_{-} s}=R * \cos (\text { lat }) * \sin (\text { lon }) \\
R_{z_{-} s}=R * \sin (\text { lat }) \\
R_{s}=\left[\begin{array}{l}
R_{x_{-} s} \\
R_{y_{-} s} \\
R_{z_{-} s}
\end{array}\right]
\end{gathered}
$$

$R_{S}$ Position vector at space

$$
\begin{gathered}
R_{w}=R * \cos (\text { lat }) * \text { Omega } \\
X_{x g}=x_{f} * \cos (\psi) \\
X_{y g}=x_{f} * \sin (\psi) \\
X_{z g}=y_{f} \\
X_{g}=\left[\begin{array}{c}
X_{x g} \\
X_{y g} \\
X_{z g}
\end{array}\right]
\end{gathered}
$$

$X_{g}$ Position vector at the end of ascent phase in ground coordinates

$$
\begin{gathered}
V_{z g}=v_{f} * \sin (\text { gammaf }) \\
V_{x g}=v_{f} * \cos (\text { gammaf }) * \cos (\psi)+R_{w} \\
V_{y g}=v_{f} * \cos (\text { gammaf }) * \sin (\psi) \\
V_{g}=\left[\begin{array}{c}
V_{x g} \\
V_{y g} \\
V_{z g}
\end{array}\right]
\end{gathered}
$$

$V_{g}$ Velocity vector at the end of ascent phase in ground coordinates

$$
r=\mathrm{C}_{s g} * X_{g}+R_{s}
$$

$r$ Position vector at the end of ascent phase in space coordinates

$$
v=\mathrm{C}_{s g} * V_{g}
$$

$v$ Velocity vector at the end of ascent phase in space coordinates

\section{PROBlem Statement}

The hypersonic vehicle is launched from the ground through a liquid rocket motor booster starting for ascent phase to altitude about $60 \mathrm{Km}$. After booster separations it started the second stage for gliding and insertion to specific orbit to higher altitude $160 \mathrm{Km}$. It continues cruise until it is inserted to the specific orbit. The objective is to maximize velocity of the vehicle; which also necessitates optimal use of 
fuel in ascent and cruise phase and also linked the independent phases together. Upon The initial conditions for ascent phase are shown in Table I.

TABLE I: INITIAL CONDITIONS

\begin{tabular}{|c|c|c|}
\hline Parameter & Symbol & value \\
\hline Downrange & $x_{0}$ & $0 \mathrm{~m}$ \\
\hline Height & $h_{0}$ & $2 \mathrm{~m}$ \\
\hline velocity & $v_{0}$ & $30 \mathrm{~m} / \mathrm{s}$ \\
\hline Flight path angle & $\gamma_{0}$ & $90^{\circ}$ \\
\hline mass & $m_{0}$ & $154200 \mathrm{Kg}$ \\
\hline Angle of attack & $\alpha_{0}$ & $0^{\circ}$ \\
\hline
\end{tabular}

\section{Gauss Pseudospectral Method Formulation}

The theory of GPM is discussed in details by David Benson [10]. GPM transforms the optimal control problem into an NLP problem which is then solved using NLP solver. The principle of Gauss pseudospectral method discretizes the state and control variables of the dynamics equations at Legendre-Gauss (LG) points. Then differentiate the polynomials to approximate the derivative of the state variables, and convert the differential equations to algebraic equations constraints. The integral parts of the cost function are approximated using the Gauss quadrature. The terminal states are determined by the initial states and the Gauss quadrature. After the transcription above, the optimal control problems are converted to nonlinear program problems with a series of algebraic equations constraints[8], [13].

$$
J=\phi\left(x(-1), t_{0}, x(1), t_{f}\right)+\frac{t_{f}-t_{0}}{2} \int_{-1}^{1} g\left(x(\tau), u(\tau), \tau ; t_{0}, t_{f}\right) d
$$

Subjected to:

$$
\begin{gathered}
\frac{d x}{d \tau}=\frac{t_{f}-t_{0}}{2} f\left(x(\tau), u(\tau), \tau ; t_{0}, t_{f}\right) \\
\Phi\left(x(-1), t_{0}, x(1), t_{f}\right)=0 \\
C\left(x(\tau), u(\tau), \tau ; t_{0}, t_{f}\right) \leq 0
\end{gathered}
$$

where $x(\tau) \in R^{n}, u(\tau) \in R^{m}$ are the state and control variables respectively; $t_{0}, t_{f}$ are the initial and final time respectively. The optimal control problem for the above equations called continues Bolza problem where time variable $\tau \in[-1,1], t \in\left[t_{0}, t_{f}\right]$ can be transformed by the following equation:

$$
t=\frac{t_{f}-t_{0}}{2} \tau+\frac{t_{f}+t_{0}}{2}
$$

Then the state variable and control variable are discretized by using a basis of $\mathrm{N}+1$ Lagrange interpolating polynomial $\mathcal{L}_{i}$ and a basis of $\mathrm{N}$ Lagrange interpolating polynomial $\mathcal{L}_{i}^{*}$ then transcribed to a nonlinear programming problem.

$$
x(\tau) \approx X(\tau)=\sum_{i=0}^{N} X\left(\tau_{i}\right) \mathcal{L}_{i}\left(\tau_{i}\right)
$$

where $\mathcal{L}_{i}(\tau)(i=0, \ldots \ldots, N)$ defined as:

$$
\mathcal{L}_{i}(\tau)=\prod_{j=0, j \neq i}^{N} \frac{\tau-\tau_{j}}{\tau_{i}-\tau_{j}}
$$

$$
\begin{gathered}
u(\tau) \approx U(\tau)=\sum_{i=1}^{N} U\left(\tau_{i}\right) \mathcal{L}_{i}^{*}\left(\tau_{i}\right) \\
\mathcal{L}_{i}^{*}(\tau)=\prod_{j=1, j \neq i}^{N} \frac{\tau-\tau_{j}}{\tau_{i}-\tau_{j}}
\end{gathered}
$$

Equations (31) and (32) satisfy the properties

$$
\begin{gathered}
\mathcal{L}_{i}(\tau)=\left\{\begin{array}{l}
1, i=j \\
0, i \neq j
\end{array}\right. \\
\mathcal{L}_{i}^{*}(\tau)=\left\{\begin{array}{l}
1, i=j \\
0, i \neq j
\end{array}\right.
\end{gathered}
$$

Differentiating the expression in Eq.(29) we get

$$
\dot{x}(\tau) \approx \dot{X}(\tau)=\sum_{i=0}^{N} X\left(\tau_{i}\right) \dot{\mathcal{L}}_{i}\left(\tau_{i}\right)
$$

The Differential approximation matrix $D \in R^{N \times N+1}$ gets the derivative of each Lagrange polynomial at Legendre Gauss (LG) points as follow

$$
D_{k i}=\dot{\mathcal{L}}_{i}\left(\tau_{k}\right)=\sum_{l=0}^{N} \frac{\prod_{j=0, j \neq i}^{N}\left(\tau_{k}-\tau_{j}\right)}{\prod_{j=0, j \neq i}^{N}\left(\tau_{i}-\tau_{j}\right)}
$$

where $k=1, \ldots, N$ and $i=0, \ldots . N$ the dynamic constraint is transcribed into algebraic constraints using the differential approximation matrix by;

$$
\begin{gathered}
\sum_{i=0}^{N} D_{k i} X_{i}-\frac{t_{f}-t_{0}}{2} f\left(x(\tau), u(\tau), \tau ; t_{0}, t_{f}\right)=0(k= \\
1, \ldots, N)
\end{gathered}
$$

where $\quad X_{k} \equiv X\left(t_{k}\right) \in R^{n} \quad$ and $\quad U_{k} \equiv U\left(t_{k}\right) \in R^{m}(k=$ $1, \ldots \ldots, N)$. The dynamic constraint is collocated only at the LG points and not at the boundary points.

Additional variables in the discretization are defined as follows, $X_{0} \equiv X(-1)$, and $X_{f} \equiv X(1)$ where $X_{f}$ is defined via the Gauss quadrature:

$$
X_{f}=X_{0}+\frac{t_{f}-t_{0}}{2} \sum_{k=1}^{N} \omega_{k} f\left(x_{k}, u_{k}, \tau_{k} ; t_{0}, t_{f}\right)
$$

where $\omega_{k}$ are the Gauss weights. The continuous cost function of Eq.(24) is approximated using a Gauss quadrature as

$$
J=\phi\left(X_{0}, t_{0}, X_{f}, t_{f}\right)+\frac{t_{f}-t_{0}}{2} \sum_{k=1}^{N} \omega_{k} g\left(x_{k}, u_{k}, \tau_{k} ; t_{0}, t_{f}\right)
$$

Finally the continuous Bolza optimal control problem is discretized as follows:

$$
\begin{gathered}
\min J=\phi\left(X_{0}, t_{0}, X_{f}, t_{f}\right)+\frac{t_{f}-t_{0}}{2} \sum_{k=1}^{N} \omega_{k} g\left(x_{k}, u_{k}, \tau_{k} ; t_{0}, t_{f}\right) \\
\text { s.t. } \sum_{i=0}^{N} D_{k i} X_{i}-\frac{t_{f}-t_{0}}{2} f\left(x(\tau), u(\tau), \tau ; t_{0}, t_{f}\right)=0 \\
\Phi\left(X_{0}, t_{0}, X_{f}, t_{f}\right)=0 \\
\mathrm{C}\left(x_{k}, u_{k}, \tau_{k} ; t_{0}, t_{f}\right) \leq 0
\end{gathered}
$$

\section{ORBITAL InSERTION METHOD FORMULATION}

Several fundamental properties of the different types of orbits are developed with the aid of the laws of conservation of angular momentum and energy. These properties include the period of elliptical orbits, the escape velocity associated with parabolic paths and the characteristic energy of hyperbolic trajectories. The specified orbit was obtained by calculating the orbital elements from the final position and velocity at the end of the ascent phase to begin the glide phase until hit the target at specified orbit. The orbital elements calculated form the position $\boldsymbol{r}$ and velocity vectors $\boldsymbol{v}$ as 
following[14]:

Inclination is dihedral angle between the orbital plane and the equatorial plane and measured according to the right-hand rule, counterclockwise around the node line vector from the equator to the orbit. . The inclination is a positive number between $0^{\circ}$ and $180^{\circ}$.

$$
i=\cos ^{-1}\left(\frac{h_{z}}{h}\right)
$$

where $h$ is Specific angular momentum,

$$
h=\boldsymbol{r} \times \boldsymbol{v}=\left[\begin{array}{ccc}
\widehat{\boldsymbol{I}} & \widehat{\boldsymbol{J}} & \widehat{\boldsymbol{K}} \\
X & Y & Z \\
v_{x} & v_{y} & v_{z}
\end{array}\right]
$$

Right ascension is the angle between the positive $X$ axis and the node line and right ascension is a positive number lying between $0^{\circ}$ and $360^{\circ}$.

$$
\begin{aligned}
& \Omega=\cos ^{-1}\left(\frac{N_{\chi}}{N}\right) \\
& \Omega= \begin{cases}\cos ^{-1}\left(\frac{N_{x}}{N}\right) & \left(N_{y} \geq 0\right) \\
360^{\circ}-\cos ^{-1}\left(\frac{N_{x}}{N}\right) & \left(N_{y}<0\right)\end{cases}
\end{aligned}
$$

$N$ defines the node line

$$
\boldsymbol{N}=\widehat{\boldsymbol{K}} \times \boldsymbol{h}=\left[\begin{array}{ccc}
\hat{\boldsymbol{I}} & \hat{\boldsymbol{J}} & \widehat{\boldsymbol{K}} \\
0 & 0 & 1 \\
h_{x} & h_{y} & h_{z}
\end{array}\right]
$$

Eccentricity, is dimensionless vector e

$$
e=\frac{1}{\mu}\left(\boldsymbol{v} \times \boldsymbol{h}-\mu \frac{\boldsymbol{r}}{r}\right)
$$

Argument of perigee, is the angle between the node line vector $\mathbf{N}$ and the eccentricity vector $\mathbf{e}$, measured in the plane of the orbit. The argument of perigee is a positive number between $0^{\circ}$ and $360^{\circ}$.

$$
\begin{gathered}
\omega=\cos ^{-1}(\text { N.e } / N e) \\
\omega= \begin{cases}\cos ^{-1}(\boldsymbol{N} \cdot \boldsymbol{e} / N e) & \left(e_{z} \geq 0\right) \\
360^{\circ}-\cos ^{-1}(\boldsymbol{N} \cdot \boldsymbol{e} / N e) & \left(e_{z}<0\right)\end{cases}
\end{gathered}
$$

True anomaly, is the angle between the eccentricity vector e and the position vector $\mathbf{r}$.

$$
\begin{gathered}
\theta=\cos ^{-1}\left(\frac{\boldsymbol{e} \cdot \boldsymbol{r}}{e r}\right) \\
\theta=\left\{\begin{array}{cc}
\cos ^{-1}\left(\frac{\boldsymbol{e} \cdot \boldsymbol{r}}{e r}\right) & \left(v_{r} \geq 0\right) \\
360^{\circ}-\cos ^{-1}\left(\frac{\boldsymbol{e} \cdot \boldsymbol{r}}{e r}\right) & \left(v_{r}<0\right)
\end{array}\right.
\end{gathered}
$$

\section{Simulation}

Trajectory optimization problem was solved using GPM. In GPM the problem was solved as a multiphase single trajectory problem using GPOPS $®[15]$ where SNOPT® is used as NLP solver.

The method presented above is applied in simulations to the trajectory optimization for ascent and glide phase flight of the hypersonic vehicle with multi-constraints. The nonlinear aerodynamic model of the hypersonic vehicle is dependent on Mach and angle of attack and the U.S. standard atmosphere. The aim of the problem is to design the endpoint the hypersonic vehicle hit the target at specific orbit.

The cost function which means minimizing the control efforts is set as

$$
J=\mathrm{T} * 0.1+10 * \dot{\alpha}_{2}^{2}+10 * \dot{\alpha}_{4}^{2}
$$

Linkage Constraints between the Phases are considered as:

1) The initial state, time of phase 2 and the final state, time of phase 1 are the same

$$
x_{0}\{2\}-x_{f}\{1\}, t_{0}\{2\}-t_{f}\{1\}=0
$$

2) We hope the velocity at phase 2 can grow up to $M=0.7 \sim 0.8$ and the angle of attack is negative.

$v_{f}\{2\}-0.8 * 325=0$

3) The initial state, time of phase 3 and the final state, time of phase 2 are the same

$$
x_{0}\{3\}-x_{f}\{2\}, t_{0}\{3\}-t_{f}\{2\}=0
$$

The initial state, time of phase 4 and the final state, time of phase 3 are the same

$$
x_{0}\{4\}-x_{f}\{3\}, t_{0}\{4\}-t_{f}\{3\}=0
$$

There are some bounds for static parameter such as:

1) Period

$$
T=\left\{\begin{array}{c}
\text { lower bound }=30 \\
\text { upper bound }=150
\end{array}\right.
$$

2) Azimuth angle

$$
\psi=\left\{\begin{array}{c}
\text { lower bound }=-p i \\
\text { upper bound }=p i
\end{array}\right.
$$

$T$ is the flying time from the end of ascent phase until hitting the target. $\psi$ is the azimuth angle. They are not specific value to let the program optimize their values while choosing the specified orbit.

There are some constraints for the orbit insertion:

$$
M_{e}=E-e * \sin (E)
$$

The final constraint and the important one are the position vectors for the hypersonic vehicle and target is equal. So in the algebraic equation form;

$$
r_{m}(t)-r_{t}(t+T)=0
$$

where $r_{m}(T)$ and $r_{t}(t+T)$ are the position vectors for the hypersonic vehicle and target varying with time respectively, and the initial position vector can be calculated from the initial orbital elements as mention of previous section.

For the hypersonic vehicle payload, we calculate the 
position from the orbital elements and the flying time as follows:

$$
\begin{gathered}
M_{m}=M_{m 0}+\left(\frac{\mu}{a_{m}^{3}}\right)^{0.5} * T \\
M_{e m}=E_{m}-e_{m} * \sin \left(E_{m}\right) \\
\theta_{m}=2 * \tan ^{-1}\left(\tan \left(\frac{E_{m}}{2}\right) *\left(\frac{1+e_{m}}{1-e_{m}}\right)^{0.5}\right) \\
p_{m}=a_{m} *\left(1-e_{m}^{2}\right) \\
r_{m}=p_{m} /\left(1+e_{m} * \cos \left(\theta_{m}\right)\right) \\
\boldsymbol{r}_{\boldsymbol{m}}=\left[\begin{array}{c}
r_{m} * \cos \left(\theta_{m}\right) \\
r_{m} * \sin \left(\theta_{m}\right) \\
0
\end{array}\right]
\end{gathered}
$$

Firstly determine the position vector over the orbit by calculate the radius $\boldsymbol{r}_{\boldsymbol{m}}$ from true anomaly $\theta_{m}$ which is calculated from mean anomaly $M_{e m}$, finally the Mean anomaly calculated from the eccentric anomaly $E_{m}$.

For the target, we calculate the position from the orbital elements as follows:

$$
\begin{gathered}
M_{t}=M_{t 0}+\left(\frac{\mu}{a_{t}^{3}}\right)^{0.5} *\left(T+t_{f}\right) \\
M_{e t}=E_{t}-e_{t} * \sin \left(E_{t}\right) \\
\theta_{t}=2 * \tan ^{-1}\left(\tan \left(\frac{E_{t}}{2}\right) *\left(\frac{1+e_{t}}{1-e_{t}}\right)^{0.5}\right) \\
p_{t}=a_{t} *\left(1-e_{t}^{2}\right) \\
r_{t}=p_{t} /\left(1+e_{t} * \cos \left(\theta_{t}\right)\right) \\
r_{t}=\left[\begin{array}{c}
r_{t} * \cos \left(\theta_{t}\right) \\
r_{t} * \sin \left(\theta_{t}\right) \\
0
\end{array}\right]
\end{gathered}
$$

The initial orbital elements:

\begin{tabular}{|c|c|c|}
\hline Parameter & Symbol & value \\
\hline $\begin{array}{c}\text { Semi-major } \\
\text { axis }\end{array}$ & $a$ & 6563749.29771536 \\
\hline eccentricity & $e$ & 0.183808345592869 \\
\hline inclination & $i$ & 0.708538550378236 \\
\hline Right ascension & $\Omega$ & 5.535561107323051 \\
\hline $\begin{array}{c}\text { Argument of } \\
\text { perigee }\end{array}$ & $\omega$ & 5.52480682348415 \\
\hline True anomaly & $\theta$ & 1.773943966073379 \\
\hline
\end{tabular}

\section{RESUlts}

In this section, Gauss pseudospectral method is utilized by GPOPS II, the MATLAB version is 2014a. The experiment is performed on Intel ${ }^{\circledR}$ core $^{\mathrm{TM}}$ i5 CPU 4.00 GB RAM. Win 10 64-bit operating System.

The following figures shows the variation for the different states includes the downrange, height, velocity, mass, flight path angle, angle of attack and finally the terminal point which the hypersonic vehicle hots the target at specific orbit. The above design procedure is used for the flight process inside and outside the dense atmosphere.

Firstly inside the dense atmosphere: Vertical flight phase $\left(0 \sim t_{1}\right)$ during this time the flight path angle equal to $90^{\circ}$ and angle of attack equal to $0^{\circ}$. Turning flight phase $\left(t_{1} \sim t_{3}\right)$ during this time, the hypersonic vehicle should complete turning. There is another time $t_{2}$ between $t_{1}$ and $t_{3}$, at this time the velocity should grow up to $\operatorname{Mach} M=0.7 \sim 0.8$ and angle of attach is negative and all the other states accomplish the desired values. From $\left(t_{2} \sim t_{3}\right)$ the hypersonic vehicle moves from the transonic speed to supersonic speed and the first stage is separated so we should keep angle of attack equal to zero to complete the separation without strong action of lateral force.

Second outside the dense atmosphere, flight in vacuum: the trajectory after separating the first stage can be considered inside vacuum for a long range to hit the target at specific orbit.

The following results show the vertical ascends to get terrain clearance after liftoff, which lasts 6 sec. thereafter ascends to insert the specified orbit. The blue, red and black lines express the vertical-rise phase while the green line express orbit-insertion phase. The following figures show the trajectory, velocity, mass, flight path angle, and angle of attack profiles respectively. Finally the last figure shows the orbit insertion profile respectively.

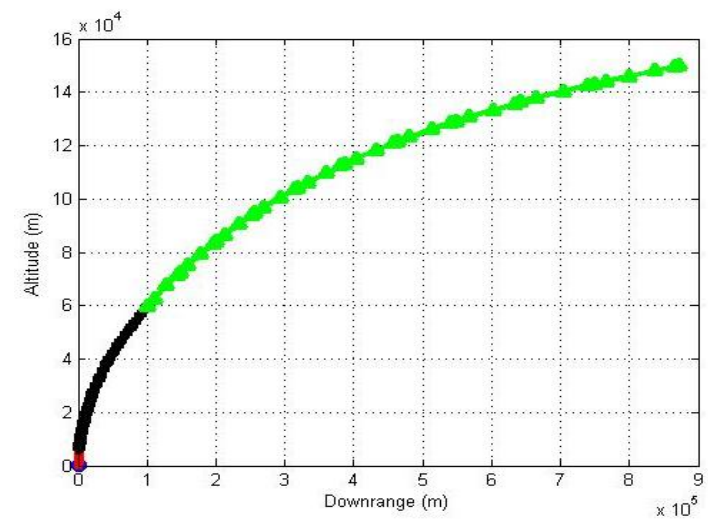

Fig. 1. Trajectory profile.

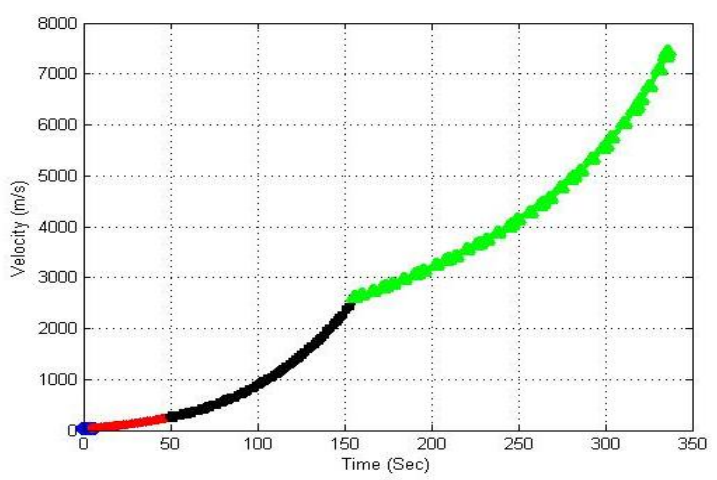

Fig. 2. Velocity profile.

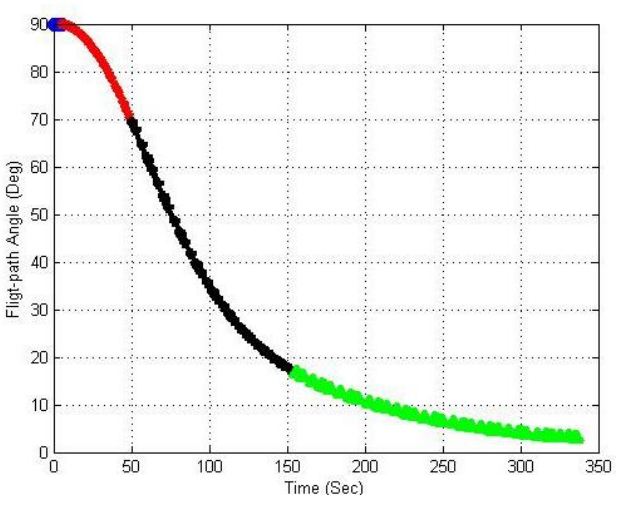

Fig. 3. Flight path angle profile. 


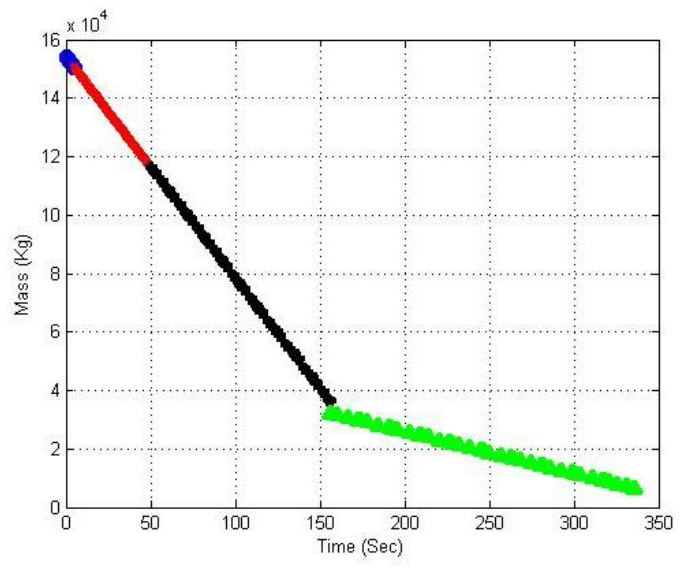

Fig. 4. Mass profile.

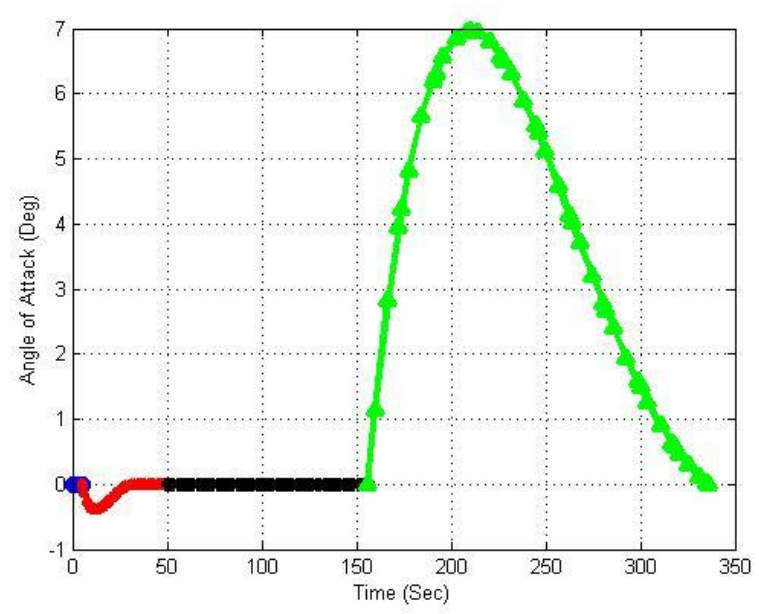

Fig. 5. Angle of Attack profile.

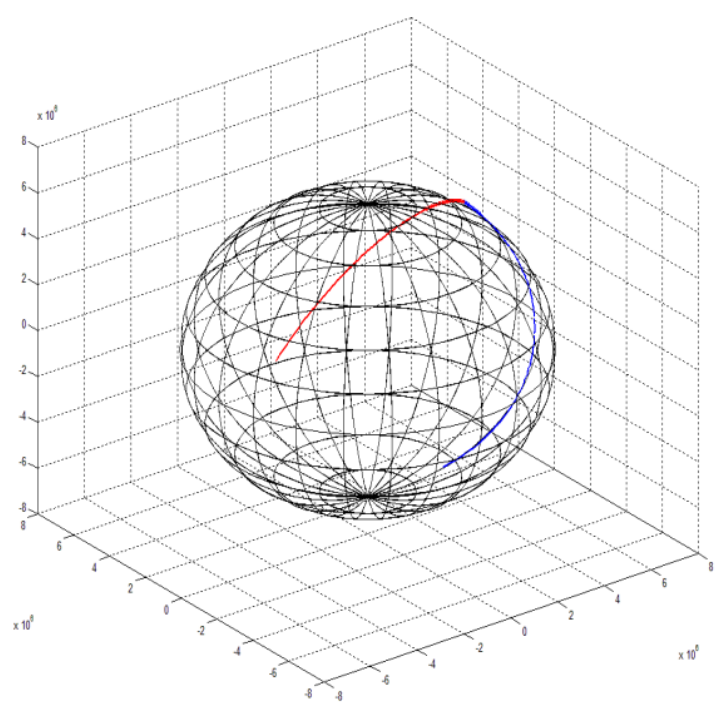

Fig. 6. Terminal point for orbit insertion.

\section{CONCLUSION}

A multi-constraints multi-phase trajectory optimization method for hypersonic vehicle has been proposed to analyze the performance for hypersonic vehicle. The models adapted for dynamics, propulsion, atmospherics, aerodynamics, have enabled to implement trajectory optimization by using
GPOPS method, a direct optimization framework to determine ascent and mid-course trajectory optimization is developed.

Trajectory optimization of hypersonic flight was carried out using GPM for an ascent-cruise profile, it presents the linkage between the phases and the endpoint functions to meets the requirements to hit the target at specific pre-described orbit.

\section{REFERENCES}

[1] D. E. Kirk, Optimal Control Theory an Introduction, Mineola, New York: Dover Publications Inc., 2004.

[2] W. Schleich, "The space shuttle ascent guidance and control," Guidance and Control Conference, San Diego,CA,U.S.A., 1982.

[3] Z. S. L. Ma, W. F. Chen, X. U. Lv, and Z. G. Song, "Three-dimensional trajectory optimization for lunar ascent using gauss pseudospectral method," presented at the AIAA Guidance, Navigation, and Control Conference, San Diego, California, USA, 2016.

[4] T. Rahman and Z. Hao, "Trajectory optimization of hypersonic vehicle using gauss and legendre pseudospectral method," presented at the Internationa Conference on Mechanical and Electrical Technology, 2011

[5] F. Fariba and R. I., "Trajectory optimization by indirect spectral collocation methods," in Proc. Astrodynamics Specialist Conference, Denver, CO, U.S.A., 2000

[6] M. A. Paluszek and S. J. Thomas, "Trajectory optimization using global methods," presented at the 29th International Electric Propulsion Conference, Princeton University, 2005.

[7] L. Y. A. N. Y. H. G. Qiang, "A survey of numerical algorithms for trajectory optimization of flight vehicles," Science China Technological Sciences, vol. 55, pp. 2538-2560, September 20122012

[8] D. Garg, "Advances in global pseudospectral methods for optimal ccontrol," Doctor of Philosophy, University of Florida, 2011.

[9] W. C. L. Ma, Z. Song, and Z. J. Shao, "A unified trajectory optimization framework for lunar ascent," Advances in Engineering Software, pp. 32-45, 2016.

[10] D. Benson, "A gauss pseudospectral transcription for optimal control," Doctor of Philosophy in Aeronautics and Astronautics, Department of Aeronautics and Astronautics, 2005.

[11] T. Rahman, "Design of guidance method for multiple phases of hypersonic vehicle flight," School of Astronautics, Beihang University, Beijing, China, Beihang University, 2014.

[12] H. Linshu, Solid Ballistic Missile Design. Beihang University, 2004.

[13] H. Y. A. C. Wanchun, "Trajectory optimization of a cruise missile using the Gauss Pseudospectral Method," presented at the Control and Decision Conference (2014 CCDC), The 26th Chinese Changsha 2014

[14] H. D. Curtis, Orbital Mechanics for Engineering Students-Aerospace Engineering, Elsevier science and technology, 2005.

[15] M. A. Patterson and A. V. Rao, "A general-purpose matlab toolbox for solving optimal control problems using the radau pseudospectral method," University of FloridaJanuary 2013.

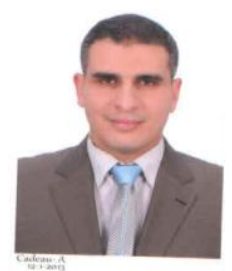

Abdel Mageed Mahmoud was born in 1981, Egyptian. He was graduated from Military Technical College in 2004. He got his master degree in 2011. Now he is a doctoral candidate school of Astronautics at Beihang University China. His research interests in guidance, navigation and control and trajectory optimization.

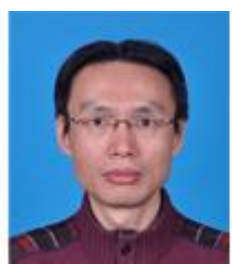

Chen wanchun was born in Nov. 1964. He received the Ph.D. degree, the M. degree, B.S. degree in the School of Astronautics from Beihang University, Beijing, China, in 1995, 1989, 1986. Since 2002, he has been a professor in the School of Astronautics, Beihang University, Beijing, China. His research interests include flight vehicle design, flight dynamics, guidance and control. 


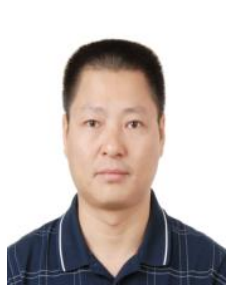

Zhou Hao was born in Sep. 1976. He received the Ph.D. degree in the School of Astronautics from Beihang University, Beijing, China, in 2006 and the B.S. degree in School of Astronautics from National University of Defense Technology, Changsha, China in 1998. Since December 2006, he has been a Lecturer in the School of Astronautics, Beihang University, Beijing, China. He was a visiting research fellow in the School of Electrical and Electronic Engineering, Nanyang Technological University, Singapore for 1 year from 2010 to 2011 His research interests include Flight Vehicle Design, Flight Dynamics, Guidance and Control.

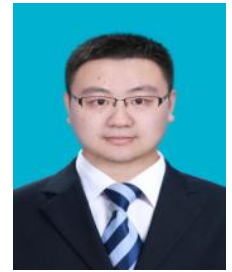

Liang Yang was born in 1985 . He is a lecturer at Beihang University. His research interests are flight mechanics, guidance and control, and trajectory optimization. 